\title{
Unsupervised Clustering Identifies Sub-Phenotypes and Reveals Pre-Dialysis Hyperlactatemia as a Novel Outcome Predictor in Dialysis-Requiring Sepsis-Associated Acute Kidney Injury
}

Chun-Fu Lai

National Taiwan University Hospital

Vin-Cent Wu ( $\sim$ q91421028@ntu.edu.tw )

National Taiwan University Hospital https://orcid.org/0000-0001-7935-0991

Jung-Hua Liu

National Chung Cheng University

Shuei-Liong Lin

National Taiwan University Hospital

Yung-Ming Chen

National Taiwan University Hospital

\section{Research}

Keywords: Acute kidney injury, Cluster analysis, Competing risk, Cohort studies, Recovery of function, Renal replacement therapy, Survival

Posted Date: November 18th, 2021

DOI: https://doi.org/10.21203/rs.3.rs-1058768/v1

License: (c) (i) This work is licensed under a Creative Commons Attribution 4.0 International License.

Read Full License 


\section{Abstract}

\section{Background}

Heterogeneity exists in sepsis-associated acute kidney injury (SA-AKI). This prospective observational cohort study aimed to perform consensus cluster analysis and investigate the clinical relevance of identified sub-phenotypes of critically ill patients with dialysis-requiring SA-AKI.

\section{Methods}

All septic patients with dialysis-requiring SA-AKI, defined by the Sepsis-3 and Kidney Disease: Improving Global Outcomes AKI criteria, admitted to an intensive care unit in Taiwan between 2002 and 2018 were included. We employed unsupervised consensus clustering based on 22 clinical variables upon initialising renal replacement therapy. They were observed until death or 90 days after hospital discharge. The outcomes were mortality and being free of dialysis.

\section{Results}

In total, 1,397 patients were enrolled (mean age of $63.8 \pm 16.38$ years and $69.7 \%$ were men). After a median follow-up period of 31 (interquartile range 8-123) days, all-cause mortality occurred in 911 patients (65.12\%). Moreover, 133 (9.51\%) survivors were dialysis dependent, where 355 (25.38\%) survivors were free of dialysis. Unsupervised consensus clustering identified three sub-phenotypes associated with significantly different risks of mortality and being free of dialysis. This strategy led us to reveal that the pre-dialysis hyperlactatemia of $\geq 3.1 \mathrm{mmol} / \mathrm{L}$ was an independent predictor of mortality and being free of dialysis according to the competing risk modeling. Our results were validated in an independent multi-center AKI cohort.

\section{Conclusions}

By the data-driven clustering analysis, we identified sub-phenotypes in septic patients with dialysisrequiring SA-AKI and revealed pre-dialysis hyperlactatemia as a novel outcome predictor. This result represents a step towards precision medicine for septic patients.

\section{Background}

Sepsis is a life-threatening syndrome characterised by multiple organ dysfunction due to a dysregulated host response to infection. Acute kidney injury (AKI) is common in sepsis [1, 2]. Sepsis is not only a leading contributing factor for AKI [3, 4], but also a predisposing factor for AKI recurrence [5]. Patients with sepsis-associated AKI (SA-AKI) have a greater burden of illness and abnormalities in haemodynamics and laboratory parameters than those with non-septic AKI [3]. We and others have shown that the presence of SA-AKI is strongly associated with increased short- and long-term mortality [3, 6]. Furthermore, among published studies on different clinical entities, $4.3-17.8 \%$ of patients with SA-AKI required renal replacement therapy $[2,4,7]$. Compared with patients who did not require dialysis, those 
who underwent dialysis during SA-AKI had a much higher risk of mortality [2] and lower possibility of kidney recovery [7]. Recent studies reported that 90-day mortality in patients with dialysis-requiring SA-AKI were ranged from $45.2-81.2 \%$ [8-10]. Therefore, this population represents the highest risk of mortality and dialysis dependence.

Recovery from AKI has been recognised as a key factor in determining clinical outcomes [5, 11-13]. However, data specifically focussing on kidney recovery after SA-AKI are limited. Two reports have shown that patients whose renal function recover after SA-AKI, even incompletely, have better survival than those who fail to recover [2, 7]. Although a standardised definition of kidney recovery is still lacking [13, 14], the development of sufficient kidney function allowing weaning off dialysis remains the most concern in patient care. We previously showed that AKI patients with septic shock were more likely to become longterm dialysis-dependent than those without sepsis [6]. In contrast, others have demonstrated that patients with SA-AKI had a higher probability of being free of dialysis than those with non-septic AKI [3, 11]. One explanation for these conflicting observations may be the competing risks of death and kidney recovery. Failure to account for competing events can lead to incorrect estimation of the effects of covariates on the outcome $[15,16]$.

Unsupervised clustering is an agnostic class discovery largely used in basic experiments and genetic studies [17]. It contributes to defining novel subtypes of study subjects and identifying previously unrecognised mechanistic pathways $[18,19]$. This data-driven approach has been successfully applied in clinical studies to identify distinct sub-phenotypes and risk factors for outcomes. It is especially valuable for investigating medical diseases with high heterogeneity, such as heart failure [20], hypertension [21], diabetes [22], chronic kidney disease [23], and AKI [24].

We hypothesised that agnostically clustering patients with dialysis-requiring SA-AKI can identify distinct subpopulations with different clinical relevance. This study was designed to examine phenotype heterogeneity in critically ill patients with dialysis-requiring SA-AKI using consensus cluster analysis of multidimensional clinical data. Understanding potential predictors of clinical outcomes may facilitate appropriate intervention and contribute to early shared decision-making for caring patients with SA-AKI.

\section{Methods}

\section{Study cohort}

The National Taiwan University Hospital Study Group on Acute Renal Failure (NSARF) database contains prospectively collected data from patients with AKI during surgical intensive care unit (ICU) admission. The details of the NSARF study have been described previously [25-28]. The study was approved by the Institutional Review Board of National Taiwan University Hospital (201407076RINA). Written informed consent was waived because there was no breach of privacy and interference with patient care.

In this observational cohort study, we investigated patients with dialysis-requiring SA-AKI, defined by the simultaneous presence of both sepsis and AKI [29]. We first screened patients aged 18 years and above 
in the database who encountered an episode of dialysis-requiring $\mathrm{AKI}$ and had suspected concurrent infection between 1 January 2002 and 31 December 2018. Dialysis-requiring AKI was defined as the need to receive renal replacement therapy (RRT) during the index AKI according to the Kidney Disease: Improving Global Outcomes AKI criteria [30]. Patients who had pre-existing end-stage kidney disease, were kidney transplant recipients or had ever received any RRT were excluded from the study. Infection was suspected as the combination of usage of systemic antibiotics and positive microbiology culture from body fluids. Sepsis was defined according to the third international consensus definition for sepsis and septic shock (Sepsis-3) [31]. To be classified as having sepsis, patients with a suspected or confirmed infection should have an acute increase in total Sequential Organ Failure Assessment (SOFA) score by $\geq 2$ or have met at least two quick SOFA criteria within the 24-h period before RRT was started. The baseline SOFA component was considered zero unless the patient has pre-existing organ dysfunction before the onset of infection [31]. Patients with chronic hepatic dysfunction and chronic respiratory impairment were assigned a baseline SOFA score of 4 and 2, respectively [32].

\section{Clinical assessments of patients and dialysis initiation}

All demographic and clinical data were prospectively collected on a standardised form [26]. The worst physiological and laboratory values during the 24-h period upon initialising RRT were collected. Serum lactate levels and body mass index were missing in 142 (10.16\%) and 47 (3.36\%) of individuals, respectively. All other variables were missing for less than $0.6 \%$. Missing data was imputed using Multivariate Imputation by Chained Equation. The doses of inotropic therapies were expressed as inotropic equivalent (IE) $[\mu \mathrm{g} / \mathrm{kg} / \mathrm{min}]$ (= dopamine + dobutamine + 100xepinephrine + 100xnorepinephrine $+100 \times$ isoprotenolol $+15 \times$ milrinone) [33]. The details of the collected parameters are shown in Table 1 and Additional file 1: Supplementary Methods. RRT was initiated according to the predetermined indications detailed in the Additional file 1: Supplementary Methods. The modality of RRT was documented as either continuous renal replacement therapy (CRRT), sustained low efficiency dialysis (SLED), or intermittent haemodialysis (iHD) according to clinical judgements.

\section{Outcomes}

This dataset also leveraged an electrical medical record to continuously recorded data for outcome analysis when patients visited our outpatient department after the index hospitalisation. The outcomes of interest upon 90 days after hospital discharge were (1) all-cause mortality and (2) being free of dialysis. All patients were followed up since the initialisation of RRT until death, 90 days after hospital discharge or 180 days after the first dialysis, whichever came first. We considered death after weaning off dialysis as mortality, rather than being free of dialysis, because biological kidney recovery without survival is not patient-centred [34].

\section{Statistical analysis}

Continuous variables were presented as mean \pm standard deviation, whereas categorical variables were presented as numbers and percentages, unless otherwise stated. To examine the heterogeneity of the patients, we applied consensus cluster analysis[23] based on 22 continuous parameters, including age, 
Charlson Comorbidity Index (CCl), baseline estimated glomerular filtration rate (eGFR), and other clinical variables upon initialising RRT (the variables shown in Table 1B). Clustering was performed using the Gower distance metric and partitioning around medoid algorithm. The optimal cluster size, ranging from 2 to 6 , was determined using consensus matrix heat maps, within-cluster consensus scores and delta area plots of the consensus cumulative distribution function (CDF) [17].

Characteristics were compared among the groups using one-way analysis of variance, the Kruskal-Wallis test, the two-sample t-test, the Mann-Whitney U-test and the chi-square test, as indicated. We plotted Kaplan-Meier curves and built multivariate Cox regression models to determine associations between patient mortality and cluster memberships and clinical variables. Since death is a competing event for kidney recovery, we performed competing risk analysis. Cluster membership and clinical variables were analysed using multivariate Fine-Gray sub-distribution hazard models for being free of dialysis. The cumulative incident function (CIF) was used to estimate the probability of being free of dialysis while treating death as a competing risk [15]. To indicate the implications of mortality against lactate levels, a generalised additive mode (GAM) incorporating subject-specific (longitudinal) random effects and adjusted for clinical factors was plotted. We defined the optimal cut-off value as the logarithm of the odds equal to zero [6]. All tests were two-tailed with significance defined by $p$ values of less than 0.05 . Statistical analyses were performed using R 4.1.0 (The R Foundation for Statistical Computing, Vienna, Austria) with the ConsensusClusterPlus package (version 1.56.0)[35] and Stata version 12 (StataCorp LLC, College Station, Texas, USA).

\section{Results}

\section{Baseline characteristics of the patients}

Among the 3,562 patients who encountered dialysis-requiring AKI and suspected infection during the study period, 1,764 were excluded due to either having a quick SOFA score of less than 2 or not having available assessment data on organ dysfunction. Of the remaining 1,798 patients, 1,397 who had an acute increase in SOFA score of 2 or higher were enrolled in the study (Fig. 1). Their mean age was 63.81 \pm 16.38 years; 973 (69.65\%) patients were male; 578 (41.37\%) patients had diabetes; and $748(53.54 \%)$ patients had hypertension. The leading sources of sepsis were respiratory tract infections $(59.99 \%)$, followed by device- or catheter-associated (26.06\%), and blood stream infections (20.47\%). (Table 1A)

\section{Table 1 Patients characteristics across the three clusters which were revealed by 22 parameters}

(A) Demographic characteristics at baseline 


\begin{tabular}{|c|c|c|c|c|c|}
\hline Variable & $\begin{array}{l}\text { Overall } \\
(n=1,397)\end{array}$ & $\begin{array}{l}\text { Cluster } 1 \\
(n=360)\end{array}$ & $\begin{array}{l}\text { Cluster } 2 \\
(n=594)\end{array}$ & $\begin{array}{l}\text { Cluster } 3 \\
(n=443)\end{array}$ & $\begin{array}{l}p \\
\text { value }^{*}\end{array}$ \\
\hline Age, years & $\begin{array}{l}63.81 \pm \\
16.38\end{array}$ & $\begin{array}{l}65.62 \pm \\
15.88\end{array}$ & $\begin{array}{l}67.98 \pm \\
15.72\end{array}$ & $\begin{array}{l}56.76 \pm \\
15.34\end{array}$ & $<0.001$ \\
\hline Male sex & $\begin{array}{l}973 \\
(69.65 \%)\end{array}$ & $\begin{array}{l}254 \\
(70.56 \%)\end{array}$ & $\begin{array}{l}408 \\
(68.69 \%)\end{array}$ & $\begin{array}{l}311 \\
(70.2 \%)\end{array}$ & 0.79 \\
\hline Current smoker or ex-smoker & $\begin{array}{l}214 \\
(15.32 \%)\end{array}$ & $\begin{array}{l}57 \\
(15.83 \%)\end{array}$ & $\begin{array}{l}74 \\
(12.46 \%)\end{array}$ & $\begin{array}{l}83 \\
(18.74 \%)\end{array}$ & 0.02 \\
\hline Diabetes mellitus & $\begin{array}{l}578 \\
(41.37 \%)\end{array}$ & $\begin{array}{l}176 \\
(48.89 \%)\end{array}$ & $\begin{array}{l}257 \\
(43.27 \%)\end{array}$ & $\begin{array}{l}145 \\
(32.73 \%)\end{array}$ & $<0.001$ \\
\hline Hypertension & $\begin{array}{l}748 \\
(53.54 \%)\end{array}$ & $\begin{array}{l}210 \\
(58.33 \%)\end{array}$ & $\begin{array}{l}340 \\
(57.24 \%)\end{array}$ & $\begin{array}{l}198 \\
(44.7 \%)\end{array}$ & $<0.001$ \\
\hline Coronary artery disease & $\begin{array}{l}418 \\
(29.92 \%)\end{array}$ & $\begin{array}{l}109 \\
(30.28 \%)\end{array}$ & $\begin{array}{l}192 \\
(32.32 \%)\end{array}$ & $\begin{array}{l}117 \\
(26.41 \%)\end{array}$ & 0.12 \\
\hline Stroke & $111(7.95 \%)$ & $33(9.17 \%)$ & $\begin{array}{l}63 \\
(10.61 \%)\end{array}$ & 15 (3.39\%) & $<0.001$ \\
\hline $\begin{array}{l}\text { Peripheral arterial occlusive } \\
\text { disease }\end{array}$ & $56(4.01 \%)$ & $26(7.22 \%)$ & $26(4.38 \%)$ & $4(0.9 \%)$ & $<0.001$ \\
\hline $\begin{array}{l}\text { Advanced heart failure, NYHA Fc } \\
\text { III or IV }\end{array}$ & $\begin{array}{l}269 \\
(19.26 \%)\end{array}$ & $\begin{array}{l}81 \\
(22.50 \%)\end{array}$ & $\begin{array}{l}97 \\
(16.33 \%)\end{array}$ & $\begin{array}{l}91 \\
(20.54 \%)\end{array}$ & 0.046 \\
\hline $\begin{array}{l}\text { Chronic obstructive pulmonary } \\
\text { disease }\end{array}$ & $72(5.15 \%)$ & $18(5 \%)$ & $42(7.07 \%)$ & $12(2.71 \%)$ & 0.007 \\
\hline Liver cirrhosis & $\begin{array}{l}177 \\
(12.67 \%)\end{array}$ & $\begin{array}{l}38 \\
(10.56 \%)\end{array}$ & $\begin{array}{l}85 \\
(14.31 \%)\end{array}$ & $\begin{array}{l}54 \\
(12.19 \%)\end{array}$ & 0.22 \\
\hline Cancer & $\begin{array}{l}295 \\
(21.12 \%)\end{array}$ & $\begin{array}{l}89 \\
(24.72 \%)\end{array}$ & $\begin{array}{l}137 \\
(23.06 \%)\end{array}$ & $\begin{array}{l}69 \\
(15.58 \%)\end{array}$ & 0.002 \\
\hline Charlson Comorbidity Index & $5.15 \pm 2.24$ & $\begin{array}{l}5.47 \pm \\
2.19\end{array}$ & $\begin{array}{l}5.72 \pm \\
2.24\end{array}$ & $4.14 \pm 1.9$ & $<0.001$ \\
\hline Baseline eGFR, $\mathrm{ml} / \mathrm{min} / 1.73 \mathrm{~m}^{2}$ & $\begin{array}{l}60.5 \pm \\
32.49\end{array}$ & $\begin{array}{l}49.47 \pm \\
33.26\end{array}$ & $\begin{array}{l}57.13 \pm \\
29.27\end{array}$ & $\begin{array}{l}73.97 \pm \\
31.47\end{array}$ & $<0.001$ \\
\hline Body mass index, $\mathrm{kg} / \mathrm{m}^{2}$ & $24.49 \pm 4.8$ & $\begin{array}{l}24.26 \pm \\
4.89\end{array}$ & $\begin{array}{l}23.92 \pm \\
4.37\end{array}$ & $\begin{array}{l}25.44 \pm \\
5.13\end{array}$ & $<0.001$ \\
\hline $\begin{array}{l}\text { Index admission year before } \\
2013\end{array}$ & $\begin{array}{l}735 \\
(52.61 \%)\end{array}$ & $\begin{array}{l}170 \\
(47.22 \%)\end{array}$ & $\begin{array}{l}334 \\
(56.23 \%)\end{array}$ & $\begin{array}{l}231 \\
(52.14 \%)\end{array}$ & 0.03 \\
\hline Surgery & $\begin{array}{l}796 \\
(56.98 \%)\end{array}$ & $\begin{array}{l}186 \\
(51.67 \%)\end{array}$ & $\begin{array}{l}346 \\
(58.25 \%)\end{array}$ & $\begin{array}{l}264 \\
(59.59 \%)\end{array}$ & 0.06 \\
\hline Total parenteral nutrition & $\begin{array}{l}328 \\
(23.48 \%)\end{array}$ & $\begin{array}{l}86 \\
(23.89 \%)\end{array}$ & $\begin{array}{l}156 \\
(26.26 \%)\end{array}$ & $\begin{array}{l}86 \\
(19.41 \%)\end{array}$ & 0.04 \\
\hline
\end{tabular}




\begin{tabular}{|c|c|c|c|c|c|}
\hline Mechanical ventilation & $\begin{array}{l}1,156 \\
(82.75 \%)\end{array}$ & $\begin{array}{l}203 \\
(56.39 \%)\end{array}$ & $\begin{array}{l}534 \\
(89.9 \%)\end{array}$ & $\begin{array}{l}419 \\
(94.58 \%)\end{array}$ & $<0.001$ \\
\hline Cardiopulmonary resuscitation & $\begin{array}{l}289 \\
(20.69 \%)\end{array}$ & $\begin{array}{l}56 \\
(15.56 \%)\end{array}$ & $\begin{array}{l}110 \\
(18.52 \%)\end{array}$ & $\begin{array}{l}123 \\
(27.77 \%)\end{array}$ & $<0.001$ \\
\hline ECMO & $\begin{array}{l}474 \\
(33.93 \%)\end{array}$ & $\begin{array}{l}74 \\
(20.56 \%)\end{array}$ & $\begin{array}{l}154 \\
(25.93 \%)\end{array}$ & $\begin{array}{l}246 \\
(55.53 \%)\end{array}$ & $<0.001$ \\
\hline \multicolumn{6}{|l|}{ Source of sepsis } \\
\hline Respiratory tract & $\begin{array}{l}838 \\
(59.99 \%)\end{array}$ & $\begin{array}{l}204 \\
(56.67 \%)\end{array}$ & $\begin{array}{l}368 \\
(61.95 \%)\end{array}$ & $\begin{array}{l}266 \\
(60.05 \%)\end{array}$ & 0.27 \\
\hline Intra-abdominal & $107(7.66 \%)$ & $32(8.89 \%)$ & $59(9.93 \%)$ & $16(3.61 \%)$ & 0.001 \\
\hline Skin or soft-tissue & $120(8.59 \%)$ & $28(7.78 \%)$ & $56(9.43 \%)$ & $36(8.13 \%)$ & 0.62 \\
\hline Genitourinary tract & $118(8.45 \%)$ & $28(7.78 \%)$ & $45(7.58 \%)$ & $\begin{array}{l}45 \\
(10.16 \%)\end{array}$ & 0.29 \\
\hline Device or catheter associated & $\begin{array}{l}364 \\
(26.06 \%)\end{array}$ & $\begin{array}{l}79 \\
(21.94 \%)\end{array}$ & $\begin{array}{l}169 \\
(28.45 \%)\end{array}$ & $\begin{array}{l}116 \\
(26.19 \%)\end{array}$ & 0.09 \\
\hline Blood stream & $\begin{array}{l}286 \\
(20.47 \%)\end{array}$ & $\begin{array}{l}69 \\
(19.17 \%)\end{array}$ & $\begin{array}{l}129 \\
(21.72 \%)\end{array}$ & $\begin{array}{l}88 \\
(19.86 \%)\end{array}$ & 0.59 \\
\hline Others / Unknown & $\begin{array}{l}406 \\
(29.06 \%)\end{array}$ & $\begin{array}{l}121 \\
(33.61 \%)\end{array}$ & $\begin{array}{l}151 \\
(25.42 \%)\end{array}$ & $\begin{array}{l}134 \\
(30.25 \%)\end{array}$ & 0.02 \\
\hline \multicolumn{6}{|l|}{ Indication for dialysis initiation } \\
\hline Azotaemia with symptoms & $\begin{array}{l}734 \\
(52.54 \%)\end{array}$ & $\begin{array}{l}222 \\
(61.67 \%)\end{array}$ & $\begin{array}{l}364 \\
(61.28 \%)\end{array}$ & $\begin{array}{l}148 \\
(33.41 \%)\end{array}$ & $<0.001$ \\
\hline Fluid overload & $623(44.6 \%)$ & $\begin{array}{l}159 \\
(44.17 \%)\end{array}$ & $\begin{array}{l}246 \\
(41.41 \%)\end{array}$ & $\begin{array}{l}218 \\
(49.21 \%)\end{array}$ & 0.04 \\
\hline Electrolyte imbalance & $155(11.1 \%)$ & $\begin{array}{l}37 \\
(10.28 \%)\end{array}$ & $56(9.43 \%)$ & $62(14 \%)$ & 0.06 \\
\hline Metabolic acidosis & $278(19.9 \%)$ & $\begin{array}{l}50 \\
(13.89 \%)\end{array}$ & $\begin{array}{l}97 \\
(16.33 \%)\end{array}$ & $\begin{array}{l}131 \\
(29.57 \%)\end{array}$ & $<0.001$ \\
\hline Oliguria & $\begin{array}{l}941 \\
(67.36 \%)\end{array}$ & $\begin{array}{l}219 \\
(60.83 \%)\end{array}$ & $\begin{array}{l}387 \\
(65.15 \%)\end{array}$ & $\begin{array}{l}335 \\
(75.62 \%)\end{array}$ & $<0.001$ \\
\hline Others & $102(7.3 \%)$ & $18(5 \%)$ & $34(5.72 \%)$ & $\begin{array}{l}50 \\
(11.29 \%)\end{array}$ & 0.001 \\
\hline Modality at the first RRT & & & & & $<0.001$ \\
\hline CRRT & $\begin{array}{l}832 \\
(59.56 \%)\end{array}$ & $\begin{array}{l}143 \\
(39.72 \%)\end{array}$ & $\begin{array}{l}320 \\
(53.87 \%)\end{array}$ & $\begin{array}{l}369 \\
(83.3 \%)\end{array}$ & \\
\hline SLED & $\begin{array}{l}274 \\
(19.61 \%)\end{array}$ & $90(25 \%)$ & $\begin{array}{l}134 \\
(22.56 \%)\end{array}$ & $\begin{array}{l}50 \\
(11.29 \%)\end{array}$ & \\
\hline $\mathrm{iHD}$ & 291 & 127 & 140 & $24(5.42 \%)$ & \\
\hline
\end{tabular}


(B) Baseline clinical data upon initialising RRT 


\begin{tabular}{|c|c|c|c|c|c|}
\hline Variable & $\begin{array}{l}\text { Overall } \\
(n=1,397)\end{array}$ & $\begin{array}{l}\text { Cluster } 1 \\
(n=360)\end{array}$ & $\begin{array}{l}\text { Cluster } 2 \\
(n=594)\end{array}$ & $\begin{array}{l}\text { Cluster } 3 \\
(n=443)\end{array}$ & $\begin{array}{l}p \\
\text { value }^{*}\end{array}$ \\
\hline $\begin{array}{l}\text { Urine volume, mL/day (median } \\
\text { [IQR]) }\end{array}$ & $\begin{array}{l}320(90- \\
768)\end{array}$ & $\begin{array}{l}420(140- \\
626.7)\end{array}$ & $\begin{array}{l}350(120- \\
833.8)\end{array}$ & $\begin{array}{l}200(50- \\
607.5)\end{array}$ & 0.005 \\
\hline Glasgow Coma Scale & $\begin{array}{l}8.24 \pm \\
4.04\end{array}$ & $\begin{array}{l}13.46 \pm \\
2.07\end{array}$ & $\begin{array}{l}7.69 \pm \\
2.21\end{array}$ & $\begin{array}{l}4.75 \pm \\
2.57\end{array}$ & $<0.001$ \\
\hline Body temperature, degree Celsius & $\begin{array}{l}36.58 \pm \\
1.15\end{array}$ & $\begin{array}{l}36.50 \pm \\
1.02\end{array}$ & $\begin{array}{l}36.65 \pm \\
1.08\end{array}$ & $\begin{array}{l}36.55 \pm \\
1.33\end{array}$ & 0.13 \\
\hline Heart rate, beats per minute & $\begin{array}{l}101.65 \pm \\
20.84\end{array}$ & $\begin{array}{l}100.59 \pm \\
21.66\end{array}$ & $\begin{array}{l}97.94 \pm \\
18.73\end{array}$ & $\begin{array}{l}107.5 \pm \\
21.58\end{array}$ & $<0.001$ \\
\hline Mean arterial pressure, $\mathrm{mmHg}$ & $\begin{array}{l}78.62 \pm \\
16.33\end{array}$ & $\begin{array}{l}82.57 \pm \\
16.1\end{array}$ & $\begin{array}{l}80.22 \pm \\
14.93\end{array}$ & $\begin{array}{l}73.27 \pm \\
16.97\end{array}$ & $<0.001$ \\
\hline $\begin{array}{l}\text { Ratio of } \mathrm{PaO} 2 \text { to fraction of } \\
\text { inspired oxygen (median [IQR]) }\end{array}$ & $\begin{array}{l}219(130- \\
356.6)\end{array}$ & $\begin{array}{l}236.4 \\
(147.9- \\
370.4)\end{array}$ & $\begin{array}{l}271 \\
(176.2- \\
395.5)\end{array}$ & $\begin{array}{l}137.7 \\
(88.5- \\
242.2)\end{array}$ & $<0.001$ \\
\hline Blood urea nitrogen, $\mathrm{mg} / \mathrm{dL}$ & $\begin{array}{l}74.05 \pm \\
44.06\end{array}$ & $\begin{array}{l}87.54 \pm \\
43.31\end{array}$ & $\begin{array}{l}80.62 \pm \\
44.08\end{array}$ & $\begin{array}{l}54.26 \pm \\
37.44\end{array}$ & $<0.001$ \\
\hline Serum creatinine, $\mathrm{mg} / \mathrm{dL}$ & $\begin{array}{l}3.75 \pm \\
2.27\end{array}$ & $4.86 \pm 2.52$ & $3.79 \pm 2.2$ & $2.8 \pm 1.65$ & $<0.001$ \\
\hline Sodium, $\mathrm{mmol} / \mathrm{L}$ & $\begin{array}{l}139.99 \pm \\
8.42\end{array}$ & $\begin{array}{l}136.78 \pm \\
7.5\end{array}$ & $\begin{array}{l}139.87 \pm \\
8.03\end{array}$ & $\begin{array}{l}142.42 \pm \\
8.79\end{array}$ & $<0.001$ \\
\hline Potassium, mmol/L & $\begin{array}{l}4.31 \pm \\
0.88\end{array}$ & $4.43 \pm 0.85$ & $\begin{array}{l}4.25 \pm \\
0.85\end{array}$ & $\begin{array}{l}4.29 \pm \\
0.95\end{array}$ & 0.008 \\
\hline White blood cells, $10^{3}$ cells $/ \mu \mathrm{L}$ & $\begin{array}{l}13.73 \pm \\
7.91\end{array}$ & $13.9 \pm 8.26$ & $\begin{array}{l}13.74 \pm \\
7.74\end{array}$ & $\begin{array}{l}13.6 \pm \\
7.86\end{array}$ & 0.86 \\
\hline Hemoglobin, g/dL & $\begin{array}{l}10.2 \pm \\
2.2\end{array}$ & $9.84 \pm 1.98$ & $\begin{array}{l}9.86 \pm \\
1.78\end{array}$ & $\begin{array}{l}10.96 \pm \\
2.64\end{array}$ & $<0.001$ \\
\hline Platelets, $10^{3}$ cells $/ \mu \mathrm{L}$ & $\begin{array}{l}126.06 \pm \\
87.95\end{array}$ & $\begin{array}{l}160.18 \pm \\
96.82\end{array}$ & $\begin{array}{l}115.79 \pm \\
80.12\end{array}$ & $\begin{array}{l}112.1 \pm \\
83.71\end{array}$ & $<0.001$ \\
\hline Total bilirubin, $\mathrm{mg} / \mathrm{dL}$ & $\begin{array}{l}4.48 \pm \\
7.42\end{array}$ & $3.15 \pm 5.8$ & $\begin{array}{l}5.25 \pm \\
8.58\end{array}$ & $\begin{array}{l}4.52 \pm \\
6.74\end{array}$ & $<0.001$ \\
\hline Bicarbonate, $\mathrm{mmol} / \mathrm{L}$ & $\begin{array}{l}19.5 \pm \\
5.32\end{array}$ & $\begin{array}{l}19.47 \pm \\
5.31\end{array}$ & $\begin{array}{l}19.53 \pm \\
5.23\end{array}$ & $\begin{array}{l}19.49 \pm \\
5.45\end{array}$ & 0.98 \\
\hline Lactate, $\mathrm{mmol} / \mathrm{L}$ & $\begin{array}{l}5.22 \pm \\
5.38\end{array}$ & $3.09 \pm 3.52$ & $\begin{array}{l}4.37 \pm \\
4.53\end{array}$ & $\begin{array}{l}8.10 \pm \\
6.39\end{array}$ & $<0.001$ \\
\hline $\begin{array}{l}\text { Inotropic equivalent, } \mu \mathrm{g} / \mathrm{kg} / \mathrm{min} \\
\text { (median [IQR]) }\end{array}$ & $\begin{array}{l}7.80(0- \\
19.70)\end{array}$ & $\begin{array}{l}1.57(0- \\
9.59)\end{array}$ & $\begin{array}{l}6.4(0- \\
16.5)\end{array}$ & $\begin{array}{l}16.2(7.4- \\
31.9)\end{array}$ & $<0.001$ \\
\hline APACH II score (median [IQR]) & $\begin{array}{l}20(16- \\
24)\end{array}$ & $15(11-19)$ & $\begin{array}{l}19(17- \\
22.8)\end{array}$ & $25(21-28)$ & $<0.001$ \\
\hline
\end{tabular}


Data are presented as mean (standard deviation), unless otherwise specified. *Variables are compared across the clusters by the one way analysis of variance, Kruskal-Wallis test, and $\chi^{2}$ test as indicated.

Abbreviations: NYHA Fc, New York Heart Association functional class; eGFR, estimated glomerular filtration rate; ECMO, extracorporeal membrane oxygenation; RRT, renal replacement therapy; CRRT, continuous renal replacement therapy; SLED, sustained low efficiency dialysis; iHD, intermittent haemodialysis; RRT, renal replacement therapy; IQR, interquartile range; APACH, Acute Physiology and Chronic Health Evaluation; SOFA, sequential organ failure assessment.

Table 1B shows the clinical data upon initialising RRT. The median IE was 7.8 (interquartile range [IQR] 019.7), median SOFA score was 13 (IQR 11-16), and the median Acute Physiology and Chronic Health Evaluation (APACH) II score was 20 (IQR 16-24). The most frequent indication for dialysis initiation was oliguria $(67.41 \%)$, followed by symptomatic azotaemia (52.54\%) and fluid overload (44.60\%). The modality of first dialysis was CRRT in $59.56 \%$, SLED in $19.61 \%$ and iHD in $20.83 \%$ of the patients.

\section{Clustering of patients with SA-AKI}

Consensus cluster analysis was performed to agnostically identify distinct subpopulations of patients. The heat maps of consensus matrix and cluster-consensus plots by different cluster sizes are shown in Additional file 1: Fig. S1A-E. Clustering with $k$ of more than 3 generated one or two clusters with a mean consensus value of less than 0.7 , indicating less stability of the cluster membership (Additional file 1: Fig. $\mathrm{S} 1 \mathrm{~F})$. The changes in the area under the CDF curve did not conspicuously increase when the cluster size $k$ was more than 3 (Additional file 1: Fig. S2). Accordingly, we identified three clusters that fairly represented the clinical parameters upon initialising RRT. Cluster 1 comprised 360 (25.77\%) patients, and cluster 2 consisted of $594(42.52 \%)$ patients, whereas cluster 3 had $443(31.71 \%)$ patients. The mean consensus value was 0.78 for cluster $1,0.72$ for cluster 2 and 0.86 for cluster 3 .

The distribution of most baseline characteristics was significantly different across the three clusters, except for sex, history of coronary artery disease, liver cirrhosis, receiving surgery, body temperature, white blood cells count and serum bicarbonate level (Table 1 and Additional file 1: Fig. S3 and S4). Fig. 2A shows the standardised difference in the baseline characteristics according to each cluster. Key features of the clusters were depicted by having an absolute standardised difference of $\geq 0.3$. Cluster 1 included individuals with poor baseline renal functional reserves, as indicated by their higher blood urea nitrogen and serum creatinine and lower baseline eGFR. However, the severity of acute illness seems to be lower, suggested by their higher Glasgow Coma Scale (GCS) and lower serum lactate, IE and APACH II and SOFA scores upon initialising RRT. In contrast, cluster 3 comprised individuals with favourable baseline conditions, that is, younger age, higher baseline eGFR and lower $\mathrm{CCl}$. Nevertheless, acute clinical status upon initialising RRT was worst in cluster 3 , as observed by their lower GCS, mean arterial pressure (MAP) and $\mathrm{PaO} 2$ to fraction of inspired oxygen ratio and higher serum lactate, IE, APACH II and SOFA scores. 
Patients in cluster 3 were more likely to receive mechanical ventilation, extracorporeal membrane oxygenation and CRRT as their first dialysis.

\section{Association between sub-phenotypes and clinical outcomes}

All enrolled patients were followed up for a median of 31 days (IQR, 8-123 days). All-cause mortality occurred in 901 (65.12\%) patients. Moreover, 139 (9.51\%) survivors were dialysis dependent, whereas 357 (25.38\%) survivors were free of dialysis. Ninety days after hospital discharge, patients in cluster 3 had the highest mortality rate (78.1\% vs. $67 \%$ [cluster 2] vs. $43.61 \%$ [cluster 1]; $p<0.001)$. Kaplan-Meier curves showed that survival differences across the three clusters were highly significant (log rank $p<0.001)$ (Fig. 2B). After adjusting for age, sex, baseline eGFR and $\mathrm{CCl}$, Cox hazard analysis showed that patients in clusters 2 (adjusted hazard ratio [HR], 1.8; 95\% Cl, 1.49-2.16) and 3 (adjusted HR, 3.06; 95\% Cl, 2.53.74) were associated with an increased risk of death compared with those in cluster 1.

The possibility of being free of dialysis 90 days after hospital discharge was highest in cluster 1 (33.89\% vs. $24.58 \%$ [cluster 2] vs. $20.09 \%$ [cluster 3]; $p<0.001$ ). The CIF plot demonstrated that the phenotypic cluster was associated with different probabilities of being free of dialysis (Gray's test $p<0.001)(F i g .2 \mathrm{C}$ ). After adjusting for age, sex, baseline eGFR and CCI in the Fine-Gray sub-distribution hazard model, patients in clusters 2 (adjusted sub-distribution hazard ratio [sHR], 0.69; 95\% $\mathrm{Cl}, 0.54-0.87$ ) and 3 (adjusted sHR, $0.4 ; 95 \% \mathrm{Cl}, 0.3-0.52$ ) were less likely to become free of dialysis than those in cluster 1.

We performed another supplementary clustering using the K-nearest neighbour graph structure and Louvain algorithm $[19,36]$. This approach identified two clusters and revealed a similar observation: a cluster featured by older age, more comorbidity and lower baseline eGFR, but with less severity of acute illness, was associated with better survival; meanwhile, another cluster featured by younger age, fewer comorbidities, higher baseline eGFR but with higher severity of acute illness was associated with an increased risk of death (Additional file 1: Fig. S5).

\section{Hyperlactatemia as a key feature of the unfavourable sub- phenotype}

Through the cluster analysis, we identified a sub-phenotype (cluster 3) of patients with a higher mortality risk and lower probability of being free of dialysis. Cluster 3 had a notably higher serum lactate level upon initialising RRT (Fig. 2A and Additional file 1: Fig. S4P). However, cluster 1, which had favourable clinical outcomes, was characterised by lower serum lactate level. This association was also evident in the supplementary clustering analyses (Additional file 1: Fig. S5).

Therefore, we examined the association of serum lactate level with clinical outcomes in this study. By applying the GAM with adjustment for age, sex, baseline eGFR, CCI and MAP upon initialising RRT, the estimated probability of mortality augmented when the serum lactate level was equal to or more than 3.1 mmol/L (Fig. 3). Baseline clinical variables were significantly different in many aspects between patients 


\section{Pre-dialysis hyperlactatemia predicts death and dialysis dependence}

Whether serum lactate levels are independently associated with mortality or kidney recovery after AKI remains controversial $[10,25,28,37,38]$. Thus, we applied a multivariable Cox proportional hazards model and included all variables listed in Table 1 to identify factors associated with mortality after dialysis-requiring SA-AKI. Serum lactate levels of $\geq 3.1 \mathrm{mmol} / \mathrm{L}$ upon initialising RRT independently predict all-cause mortality (adjusted HR, 1.23; 95\% Cl, 1.05-1.44)(Table 2). The Kaplan-Meier plot revealed that patients with hyperlactatemia of $\geq 3.1 \mathrm{mmol} / \mathrm{L}$ upon initialising RRT had poor survival (log rank $p<0.001$ )(Fig. 4A). After controlling for mortality as a competing risk and adjusting for all variables listed in Table 1, patients who had hyperlactatemia of $\geq 3.1 \mathrm{mmol} / \mathrm{L}$ upon initialising RRT were less likely to become free of dialysis (adjusted sHR, 0.74; $95 \% \mathrm{Cl}, 0.57-0.97$ )(Table 2). Moreover, the CIF plot depicted that serum lactate levels of less than $3.1 \mathrm{mmol} / \mathrm{L}$ upon initialising RRT were associated with a higher probability of being free of dialysis after dialysis-requiring SA-AKI (Gray's test $p<0.001$ )(Fig. 4B). 
Table 2

Multivariable-adjusted analysis for independent predictors for clinical outcomes.

\begin{tabular}{|c|c|c|c|c|}
\hline \multirow[t]{2}{*}{ Variables } & \multicolumn{2}{|l|}{ Mortality* } & \multicolumn{2}{|c|}{ Being free of dialysis ${ }^{\dagger}$} \\
\hline & $\mathrm{HR}^{*}, \mp(95 \% \mathrm{Cl})$ & $P$ & $\begin{array}{l}\operatorname{sHR}^{\dagger, \neq}(95 \% \\
\text { Cl) }\end{array}$ & $P$ \\
\hline Age, years & $1.02(1.01-1.02)$ & $<0.001$ & & ns \\
\hline Chronic obstructive pulmonary disease & & ns & $\begin{array}{l}1.84(1.09- \\
3.09)\end{array}$ & 0.02 \\
\hline Cancer & $1.46(1.19-1.79)$ & $<0.001$ & $\begin{array}{l}0.69(0.49- \\
0.96)\end{array}$ & 0.03 \\
\hline Index admission year before 2013 & $2.03(1.69-2.43)$ & $<0.001$ & $\begin{array}{l}0.56(0.42- \\
0.74)\end{array}$ & $<0.001$ \\
\hline Baseline eGFR, $\mathrm{ml} / \mathrm{min} / 1.73 \mathrm{~m}^{2}$ & & ns & $\begin{array}{l}1.006(1.002- \\
1.01)\end{array}$ & 0.004 \\
\hline Surgery & $0.76(0.65-0.89)$ & $<0.001$ & $\begin{array}{l}1.37(1.07- \\
1.74)\end{array}$ & 0.01 \\
\hline $\begin{array}{l}\text { Dialysis initiation due to symptomatic } \\
\text { azotaemia }\end{array}$ & $0.8(0.68-0.94)$ & 0.008 & & ns \\
\hline $\log ($ urine volume, $\mathrm{mL} /$ day) & & ns & $\begin{array}{l}1.12(1.04- \\
1.22)\end{array}$ & 0.004 \\
\hline Glasgow Coma Scale & $0.95(0.92-0.98)$ & 0.001 & $\begin{array}{l}1.05(1.001- \\
1.11)\end{array}$ & 0.046 \\
\hline Body temperature, degree Celsius & $0.92(0.87-0.98)$ & 0.01 & & ns \\
\hline Mean arterial pressure, $\mathrm{mmHg}$ & $0.99(0.99-0.99)$ & $<0.001$ & $\begin{array}{l}1.01(1.003- \\
1.02)\end{array}$ & 0.005 \\
\hline Blood urea nitrogen, $\mathrm{mg} / \mathrm{dL}$ & $\begin{array}{l}1.004(1.002- \\
1.006)\end{array}$ & $<0.001$ & & ns \\
\hline
\end{tabular}

*Multivariable Cox regression analysis of hazard ratios (HR) for mortality.

${ }^{\dagger}$ Multivariable Fine-Gray model was used, higher sub-distribution hazard ratio (sHR) implies higher probability of kidney recovery from dialysis, taking mortality as a competing risk.

¥Other factors included within both models which are not statistically significant: sex, smoking, diabetes, hypertension, coronary artery disease, stroke, peripheral arterial occlusive disease, advanced heart failure, liver cirrhosis, Charlson Comorbidity Index, body mass index, total parenteral nutrition, mechanical ventilation, cardiopulmonary resuscitation, extracorporeal membrane oxygenation, origin of sepsis, indications of dialysis initiations other than symptomatic azotaemia, modality at the first RRT, heart rate, ratio of $\mathrm{PaO} 2$ to fraction of inspired oxygen, potassium, white blood cells count, inotropic equivalent, and APACH II score.

Abbreviations: Cl: confidence interval; ns: not significant; eGFR, estimated glomerular filtration rate. 


\begin{tabular}{|c|c|c|c|c|}
\hline \multirow[t]{2}{*}{ Variables } & \multicolumn{2}{|l|}{ Mortality $^{*}$} & \multicolumn{2}{|c|}{ Being free of dialysis ${ }^{\dagger}$} \\
\hline & $\mathrm{HR}^{*}, \mp(95 \% \mathrm{Cl})$ & $P$ & $\begin{array}{l}\left.\operatorname{sHR}_{\mathrm{Cl}}\right)^{\dagger \neq \ddagger}(95 \% \\
\end{array}$ & $P$ \\
\hline Serum creatinine, $\mathrm{mg} / \mathrm{dL}$ & $0.89(0.85-0.94)$ & $<0.001$ & & ns \\
\hline Sodium, $\mathrm{mmol} / \mathrm{L}$ & $\begin{array}{l}1.01(1.003- \\
1.02)\end{array}$ & 0.01 & & ns \\
\hline Hemoglobin, g/dL & & ns & $\begin{array}{l}1.07(1.007- \\
1.13)\end{array}$ & 0.03 \\
\hline Platelets, $10^{3}$ cells $/ \mu \mathrm{L}$ & $0.999(0.998-1)$ & 0.02 & $\begin{array}{l}1.002(1- \\
1.003)\end{array}$ & 0.02 \\
\hline Total bilirubin, mg/dL & $\begin{array}{l}1.02(1.01- \\
1.031)\end{array}$ & $<0.001$ & $\begin{array}{l}0.96(0.94- \\
0.99)\end{array}$ & 0.002 \\
\hline Bicarbonate, $\mathrm{mmol} / \mathrm{L}$ & & ns & $\begin{array}{l}0.97(0.94- \\
0.995)\end{array}$ & 0.03 \\
\hline Lactate $\geq 3.1 \mathrm{mmol} / \mathrm{L}$ & $1.23(1.05-1.44)$ & 0.01 & $\begin{array}{l}0.74(0.57- \\
0.97)\end{array}$ & 0.03 \\
\hline SOFA score & $1.06(1.02-1.1)$ & 0.004 & & ns \\
\hline \multicolumn{5}{|c|}{ *Multivariable Cox regression analysis of hazard ratios (HR) for mortality. } \\
\hline \multicolumn{5}{|c|}{$\begin{array}{l}{ }^{\dagger} \text { Multivariable Fine-Gray model was used, higher sub-distribution hazard ratio (sHR) implies higher } \\
\text { probability of kidney recovery from dialysis, taking mortality as a competing risk. }\end{array}$} \\
\hline \multicolumn{5}{|c|}{$\begin{array}{l}\text { ₹0ther factors included within both models which are not statistically significant: sex, smoking, } \\
\text { diabetes, hypertension, coronary artery disease, stroke, peripheral arterial occlusive disease, advanced } \\
\text { heart failure, liver cirrhosis, Charlson Comorbidity Index, body mass index, total parenteral nutrition, } \\
\text { mechanical ventilation, cardiopulmonary resuscitation, extracorporeal membrane oxygenation, origin } \\
\text { of sepsis, indications of dialysis initiations other than symptomatic azotaemia, modality at the first } \\
\text { RRT, heart rate, ratio of PaO2 to fraction of inspired oxygen, potassium, white blood cells count, } \\
\text { inotropic equivalent, and APACH II score. }\end{array}$} \\
\hline
\end{tabular}

\section{External validation}

To validate the predictive role of hyperlactatemia, we analysed a multi-centre database of the nationwide epidemiology and prognosis of dialysis-requiring AKI (NEP-AKI-D) study [6, 8]. We extracted a subset of 190 patients with dialysis-requiring SA-AKI who underwent surgery in the index admission between 2014 and 2016 (Additional file 1: Supplementary Methods, Fig. S6 and Table S2). Considering our main findings, the Kaplan-Meier curves showed that patients with hyperlactatemia of $\geq 3.1 \mathrm{mmol} / \mathrm{L}$ upon initialising RRT was associated with lower survival than those with serum lactate levels of less than 3.1 $\mathrm{mmol} / \mathrm{L}$ (log rank $p=0.002$ )(Fig. 5). After adjusting for age, sex, baseline eGFR, CCI and MAP and SOFA scores upon initialising RRT, Cox hazard analysis showed that patients who had pre-dialysis 
hyperlactatemia of $\geq 3.1 \mathrm{mmol} / \mathrm{L}$ had increased risks of death (adjusted $\mathrm{HR}, 1.6 ; 95 \% \mathrm{Cl}, 1.1-2.33$ ) (Additional file 1: Fig. S7).

\section{Discussion}

In this study, we performed consensus clustering in critically ill patients with dialysis-requiring SA-AKI and identified three clusters. The cluster membership synthesized patients' age, baseline eGFR, CCI and clinical data patterns upon initialising RRT. Importantly, the revealed sub-phenotypes showed relevant association with patient mortality and being free of dialysis. Moreover, this data-driven approach led us to reveal various independent outcome predictors, including hyperlactatemia upon initialising RRT, which is a novel outcome predictor.

\section{Clustering and sub-phenotypes}

Our results highlighted the potential of using unsupervised clustering to explore clinically meaningful subphenotypes in such a heterogeneous population of dialysis-requiring SA-AKI. The crude mortality 90 days after hospital discharge significantly increased from patients in cluster 1 to those in clusters 2 and 3 in a graded manner. By summarising patients' comorbidity profiles and acute-illness severities, the cluster membership provided a simple prediction of outcomes. Although patients in cluster 3 were younger, had lower $\mathrm{CCl}$ and had higher baseline eGFR, they had worse disease severities upon initialising RRT and poor clinical outcomes (Table 1, and Additional file 1: Fig. S3 and S4). This suggests that the impact of acuteillness severity outweighs that of baseline general condition in patients with dialysis-requiring SA-AKI.

\section{Becoming free of dialysis}

Identifying predictive factors for kidney recovery after SA-AKI contributes to the risk stratification of individuals and facilitates appropriate interventions. Note that death and kidney recovery are competing events since patients who die early have no chance to achieve kidney recovery later. Using standard Cox models may misestimate the risk of an event in the presence of a competing risk $[15,16]$. In this study, we used the Fine-Gray sub-distribution model to estimate the effects of variables on being free of dialysis with death as the competing risk. For comparison, the CIF curve of kidney recovery from dialysis by competing risk analysis and the corresponding naïve incidence function (Kaplan-Meier curve) were overlaid in Additional file 1: Fig. S8. This demonstrates that not accounting for the competing risk of mortality overestimates the incidence of being free of dialysis.

\section{Hyperlactatemia and outcomes}

Another advantage of unsupervised clustering is its potential to discover previously unrecognised or unconfirmed predictive factors. Although hyperlactatemia of $>2 \mathrm{mmol} / \mathrm{L}$ is criterion of septic shock according to the Sepsis-3, it is not mandated as a criterion of sepsis [31]. It has been reported that hyperlactatemia is an independent mortality predictor in patients with SA-AKI in the emergency 
department [38], postoperative AKI requiring RRT [25] and SA-AKI requiring CRRT [10]. However, other studies have shown that the initial lactate level is not independently associated with death in patients with SA-AKI requiring CRRT [37] or post-cardiovascular surgery AKI [28]. In the literature, serum lactate has never been reported as an independent predictor of kidney recovery after $\operatorname{AKI}[5,7,13,25,39]$. In this study, consensus clustering demonstrated that serum lactate levels upon initialising RRT were a disparate feature among clusters (Fig. 2A). Patients with serum lactate levels of $\geq 3.1 \mathrm{mmol} / \mathrm{L}$ upon initialising RRT had not only a higher risk of mortality but also a higher risk of dialysis dependence, independent of blood pressure, the dose of vasoactive agents and disease severity (Table 2). Note that hyperlactatemia at a cut-off of $3.1 \mathrm{mmol} / \mathrm{L}$, rather than the traditional threshold of $2 \mathrm{mmol} / \mathrm{L}$, provides superior information on prognosis in patients with dialysis-requiring SA-AKI. Whether hyperlactatemia of $\geq 3.1$ $\mathrm{mmol} / \mathrm{L}$ should be considered an indication of dialysis initiation or surrogate of disease severity for SAAKI requires further investigations.

This novel finding has some possible explanations. First, metabolic reprogramming is a fundamental mechanism in developing SA-AKI. Accumulating evidence suggests that hyperlactatemia in sepsis is more explained by the accelerated aerobic glycolysis in the injured renal tubular cells and recruited leukocyte [40-42]. Animal experiments of AKI have demonstrated that lactate is overproduced in renal content and efflux into systemic circulation [43,44]. Moreover, human studies have shown that serum lactate level is significantly higher in septic patients with AKI than those without AKI [38,45]. Based on these pieces of evidence, an increased serum lactate level indicates a higher severity of sepsis and injury to the kidneys. During successful renal tubule recovery, cells may inhibit aerobic glycolysis and restore oxidative phosphorylation in the mitochondria [42]. This phasic shift requires functional mitochondria pools. Studies have shown that renal tubules that fail to recover are characterised by progressive mitochondrial pathology and persistently enhanced aerobic glycolysis [42, 44]. Patients with a higher serum lactate levels may represent more significant mitochondrial injury and less kidney reserve, resulting in a lower probability of functional recovery.

\section{Study limitation}

This study has some limitations. First, this study was conducted in the same ethnical ICU, although we have validated the results in an independent multi-centre dataset. Whether our results are generalisable to other AKI populations requires further validation. Second, the observational nature of this study without a pre-specified protocol of intervention cannot conclude the causal relationship. Third, our data-driven cluster analysis approach directly relied on the input of the data. Using different inclusive variables may result in discrete sub-phenotypes. Lastly, the clustering approach was conducted upon RRT initiation to allow the application of this study to clinical practice. Whether sub-phenotypes of patients and/or predialysis hyperlactatemia would permit earlier intervention and the mitigation of mortality is unclear and requires further evaluations.

\section{Conclusions}


The mortality rate in patients with dialysis-requiring SA-AKI was high, whereas more than a quarter of them survived and were free of dialysis 90 days after hospital discharge. Deploying unsupervised consensus clustering in this heterogeneous population could segregate them into distinct subphenotypes featured with different clinical manifestations and outcomes. We discovered that serum lactate levels of $3.1 \mathrm{mmol} / \mathrm{L}$ or more upon initialising RRT are a novel prognostic factor for risk of death and probability of achieving dialysis-free status. Although external validation is necessary, this approach may help to tailor patient-centred care and move towards precision medicine.

\section{Declarations}

\section{Ethics approval and consent to participate}

This study was approved by the Institutional Review Board of the National Taiwan University Hospital, Taipei, Taiwan (201407076RINA). An informed consent was waived because there was no breach of privacy and on interference with patient care.

\section{Consent for publication}

Not applicable.

\section{Availability of data and materials}

The datasets used and analysed during the current study are available upon reasonable request to the corresponding author, V.C. Wu.

\section{Competing interests}

The authors declare that they have no competing interests.

\section{Funding}

This work was supported by the Ministry of Science and Technology, Taiwan (MOST 107-2314-B-002177-MY3, 107-2314-B-002-026-MY3, 108-2314-B-002-058, 109-2314-B-002-174-MY3), National Health Research Institutes, Taiwan (PH 102-SP-09), National Taiwan University Hospital (108-N06, 109-S4634, PC-1264, PC-1309, VN109-09, UN109-041, UN110-030, 110C101-074, 110-S4889), Ministry of Health and Welfare, Taiwan (MOHW 110-TDU-B-212-124005), and Mrs. Hsiu-Chin Lee Kidney Research Fund. The funders had no role in the design of the study and collection, analysis, and interpretation of data and in wiring the manuscript.

\section{Authors' contributions}

Concept and design: C.F. Lai, V.C. Wu. Acquisition, analysis, and interpretation of data: All authors. Drafting of the manuscript: C.F. Lai, V.C. Wu. Critical revision of the manuscript for important intellectual 
content: J.H. Liu, S.L. Lin, Y.M. Chen. Obtained funding and study supervision: C.F. Lai, V.C. Wu. All authors have seen the final version of manuscript, endorse the data, and approve the conclusions.

\section{Acknowledgements}

We thank Miss Fang-Yu Yeh for her assistant in statistical analysis. We also thank the staff of the Second and Seventh Core Labs, Department of Medical Research at National Taiwan University Hospital for technical assistance.

\section{References}

1. Vincent JL, Sakr Y, Sprung CL, Ranieri VM, Reinhart K, Gerlach H, et al. Sepsis in European intensive care units: results of the SOAP study. Crit Care Med. 2006;34:344-53.

2. Kellum JA, Chawla LS, Keener C, Singbartl K, Palevsky PM, Pike FL, et al. The Effects of Alternative Resuscitation Strategies on Acute Kidney Injury in Patients with Septic Shock. Am J Respir Crit Care Med. 2016;193:281-7.

3. Bagshaw SM, Uchino S, Bellomo R, Morimatsu H, Morgera S, Schetz M, et al. Septic acute kidney injury in critically ill patients: clinical characteristics and outcomes. Clin J Am Soc Nephrol. 2007;2:431-9.

4. Shum HP, Kong HH, Chan KC, Yan WW, Chan TM. Septic acute kidney injury in critically ill patients - a single-center study on its incidence, clinical characteristics, and outcome predictors. Ren Fail. 2016;38:706-16.

5. Kellum JA, Sileanu FE, Bihorac A, Hoste EA, Chawla LS. Recovery after Acute Kidney Injury. Am J Respir Crit Care Med. 2017;195:784-91.

6. Wu VC, Chueh SJ, Chang JT, Hsu BG, Ostermann M, Chu TS. Acute Kidney Injury and Septic ShockDefined by Updated Sepsis-3 Criteria in Critically III Patients. J Clin Med. 2019;8.

7. Fiorentino M, Tohme FA, Wang S, Murugan R, Angus DC, Kellum JA. Long-term survival in patients with septic acute kidney injury is strongly influenced by renal recovery. PLoS One. 2018;13:e0198269.

8. Chen YY, Wu VC, Huang WC, Yeh YC, Wu MS, Huang CC, et al. Norepinephrine Administration Is Associated with Higher Mortality in Dialysis Requiring Acute Kidney Injury Patients with Septic Shock. J Clin Med. 2018;7.

9. Barbar SD, Clere-Jehl R, Bourredjem A, Hernu R, Montini F, Bruyere R, et al. Timing of RenalReplacement Therapy in Patients with Acute Kidney Injury and Sepsis. N Engl J Med. 2018;379:1431-42.

10. Jarvisalo MJ, Hellman T, Uusalo P. Mortality and associated risk factors in patients with blood culture positive sepsis and acute kidney injury requiring continuous renal replacement therapy-A retrospective study. PLoS One. 2021;16:e0249561.

11. Hickson LJ, Chaudhary S, Williams AW, Dillon JJ, Norby SM, Gregoire JR, et al. Predictors of outpatient kidney function recovery among patients who initiate hemodialysis in the hospital. Am J 
Kidney Dis. 2015;65:592-602.

12. Cerda J, Liu KD, Cruz DN, Jaber BL, Koyner JL, Heung M, et al. Promoting Kidney Function Recovery in Patients with AKI Requiring RRT. Clin J Am Soc Nephrol. 2015;10:1859-67.

13. Forni LG, Darmon M, Ostermann M, Oudemans-van Straaten HM, Pettila V, Prowle JR, et al. Renal recovery after acute kidney injury. Intensive Care Med. 2017;43:855-66.

14. Chawla LS, Bellomo R, Bihorac A, Goldstein SL, Siew ED, Bagshaw SM, et al. Acute kidney disease and renal recovery: consensus report of the Acute Disease Quality Initiative (ADQI) 16 Workgroup. Nat Rev Nephrol. 2017;13:241-57.

15. Austin PC, Lee DS, Fine JP. Introduction to the Analysis of Survival Data in the Presence of Competing Risks. Circulation. 2016;133:601-9.

16. Ravani P, Fiocco M, Liu P, Quinn RR, Hemmelgarn B, James M, et al. Influence of Mortality on Estimating the Risk of Kidney Failure in People with Stage 4 CKD. J Am Soc Nephrol. 2019;30:221927.

17. Monti S, Tamayo P, Mesirov J, Golub T. Consensus Clustering: A Resampling-Based Method for Class Discovery and Visualization of Gene Expression Microarray Data. Machine Learning. 2003;52:91118.

18. Park J, Shrestha R, Qiu C, Kondo A, Huang S, Werth M, et al. Single-cell transcriptomics of the mouse kidney reveals potential cellular targets of kidney disease. Science. 2018;360:758-63.

19. Wu H, Kirita Y, Donnelly EL, Humphreys BD. Advantages of Single-Nucleus over Single-Cell RNA Sequencing of Adult Kidney: Rare Cell Types and Novel Cell States Revealed in Fibrosis. J Am Soc Nephrol. 2019;30:23-32.

20. Ahmad T, Pencina MJ, Schulte PJ, O'Brien E, Whellan DJ, Pina IL, et al. Clinical implications of chronic heart failure phenotypes defined by cluster analysis. J Am Coll Cardiol. 2014;64:1765-74.

21. Guo Q, Lu X, Gao Y, Zhang J, Yan B, Su D, et al. Cluster analysis: a new approach for identification of underlying risk factors for coronary artery disease in essential hypertensive patients. Sci Rep. 2017;7:43965.

22. Ahlqvist E, Storm P, Karajamaki A, Martinell M, Dorkhan M, Carlsson A, et al. Novel subgroups of adult-onset diabetes and their association with outcomes: a data-driven cluster analysis of six variables. Lancet Diabetes Endocrinol. 2018;6:361-9.

23. Zheng Z, Waikar SS, Schmidt IM, Landis JR, Hsu CY, Shafi T, et al. Subtyping CKD Patients by Consensus Clustering: The Chronic Renal Insufficiency Cohort (CRIC) Study. J Am Soc Nephrol. 2021;32:639-53.

24. Chaudhary K, Vaid A, Duffy A, Paranjpe I, Jaladanki S, Paranjpe M, et al. Utilization of Deep Learning for Subphenotype Identification in Sepsis-Associated Acute Kidney Injury. Clin J Am Soc Nephrol. 2020;15:1557-65.

25. Lin YF, Ko WJ, Chu TS, Chen YS, Wu VC, Chen YM, et al. The 90-day mortality and the subsequent renal recovery in critically ill surgical patients requiring acute renal replacement therapy. Am J Surg. 2009;198:325-32. 
26. Wu VC, Huang TM, Lai CF, Shiao CC, Lin YF, Chu TS, et al. Acute-on-chronic kidney injury at hospital discharge is associated with long-term dialysis and mortality. Kidney Int. 2011;80:1222-30.

27. Lai CF, Wu VC, Huang TM, Yeh YC, Wang KC, Han YY, et al. Kidney function decline after a nondialysis-requiring acute kidney injury is associated with higher long-term mortality in critically ill survivors. Crit Care. 2012;16:R123.

28. Shiao CC, Huang YT, Lai TS, Huang TM, Wang JJ, Huang CT, et al. Perioperative body weight change is associated with in-hospital mortality in cardiac surgical patients with postoperative acute kidney injury. PLoS One. 2017;12:e0187280.

29. Bellomo R, Kellum JA, Ronco C, Wald R, Martensson J, Maiden M, et al. Acute kidney injury in sepsis. Intensive Care Med. 2017;43:816-28.

30. Levey AS, Eckardt KU, Dorman NM, Christiansen SL, Cheung M, Jadoul M, et al. Nomenclature for Kidney Function and Disease: Executive Summary and Glossary from a Kidney Disease: Improving Global Outcomes (KDIGO) Consensus Conference. Transpl Int. 2020.

31. Singer M, Deutschman CS, Seymour CW, Shankar-Hari M, Annane D, Bauer M, et al. The Third International Consensus Definitions for Sepsis and Septic Shock (Sepsis-3). JAMA. 2016;315:80110.

32. Raith EP, Udy AA, Bailey M, McGloughlin S, Maclsaac C, Bellomo R, et al. Prognostic Accuracy of the SOFA Score, SIRS Criteria, and qSOFA Score for In-Hospital Mortality Among Adults With Suspected Infection Admitted to the Intensive Care Unit. JAMA. 2017;317:290-300.

33. Chen YS, Lin JW, Yu HY, Ko WJ, Jerng JS, Chang WT, et al. Cardiopulmonary resuscitation with assisted extracorporeal life-support versus conventional cardiopulmonary resuscitation in adults with in-hospital cardiac arrest: an observational study and propensity analysis. Lancet. 2008;372:554-61.

34. Kellum JA. How can we define recovery after acute kidney injury? Considerations from epidemiology and clinical trial design. Nephron Clin Pract. 2014;127:81-8.

35. Wilkerson MD, Hayes DN. ConsensusClusterPlus: a class discovery tool with confidence assessments and item tracking. Bioinformatics. 2010;26:1572-3.

36. Stuart T, Butler A, Hoffman P, Hafemeister C, Papalexi E, Mauck WM, 3rd, et al. Comprehensive Integration of Single-Cell Data. Cell. 2019;177:1888-902 e21.

37. Passos RDH, Ramos JGR, Gobatto A, Mendonca EJB, Miranda EA, Dutra FRD, et al. Lactate clearance is associated with mortality in septic patients with acute kidney injury requiring continuous renal replacement therapy: A cohort study. Medicine (Baltimore). 2016;95:e5112.

38. Hsu YC, Hsu CW. Septic acute kidney injury patients in emergency department: The risk factors and its correlation to serum lactate. Am J Emerg Med. 2019;37:204-8.

39. Pike F, Murugan R, Keener C, Palevsky PM, Vijayan A, Unruh M, et al. Biomarker Enhanced Risk Prediction for Adverse Outcomes in Critically III Patients Receiving RRT. Clin J Am Soc Nephrol. 2015;10:1332-9. 
40. Yang L, Xie M, Yang M, Yu Y, Zhu S, Hou W, et al. PKM2 regulates the Warburg effect and promotes HMGB1 release in sepsis. Nat Commun. 2014;5:4436.

41. Garcia-Alvarez M, Marik P, Bellomo R. Sepsis-associated hyperlactatemia. Crit Care. 2014;18:503.

42. Peerapornratana S, Manrique-Caballero CL, Gomez H, Kellum JA. Acute kidney injury from sepsis: current concepts, epidemiology, pathophysiology, prevention and treatment. Kidney Int. 2019;96:1083-99.

43. Zager RA, Johnson AC, Becker K. Renal cortical pyruvate depletion during AKI. J Am Soc Nephrol. 2014;25:998-1012.

44. Lan R, Geng H, Singha PK, Saikumar P, Bottinger EP, Weinberg JM, et al. Mitochondrial Pathology and Glycolytic Shift during Proximal Tubule Atrophy after Ischemic AKI. J Am Soc Nephrol. 2016;27:3356-67.

45. Plataki M, Kashani K, Cabello-Garza J, Maldonado F, Kashyap R, Kor DJ, et al. Predictors of acute kidney injury in septic shock patients: an observational cohort study. Clin J Am Soc Nephrol. 2011;6:1744-51.

\section{Figures}




\section{ICU patients with dialysis-requiring AKI and suspected infection $(n=3,562)$}
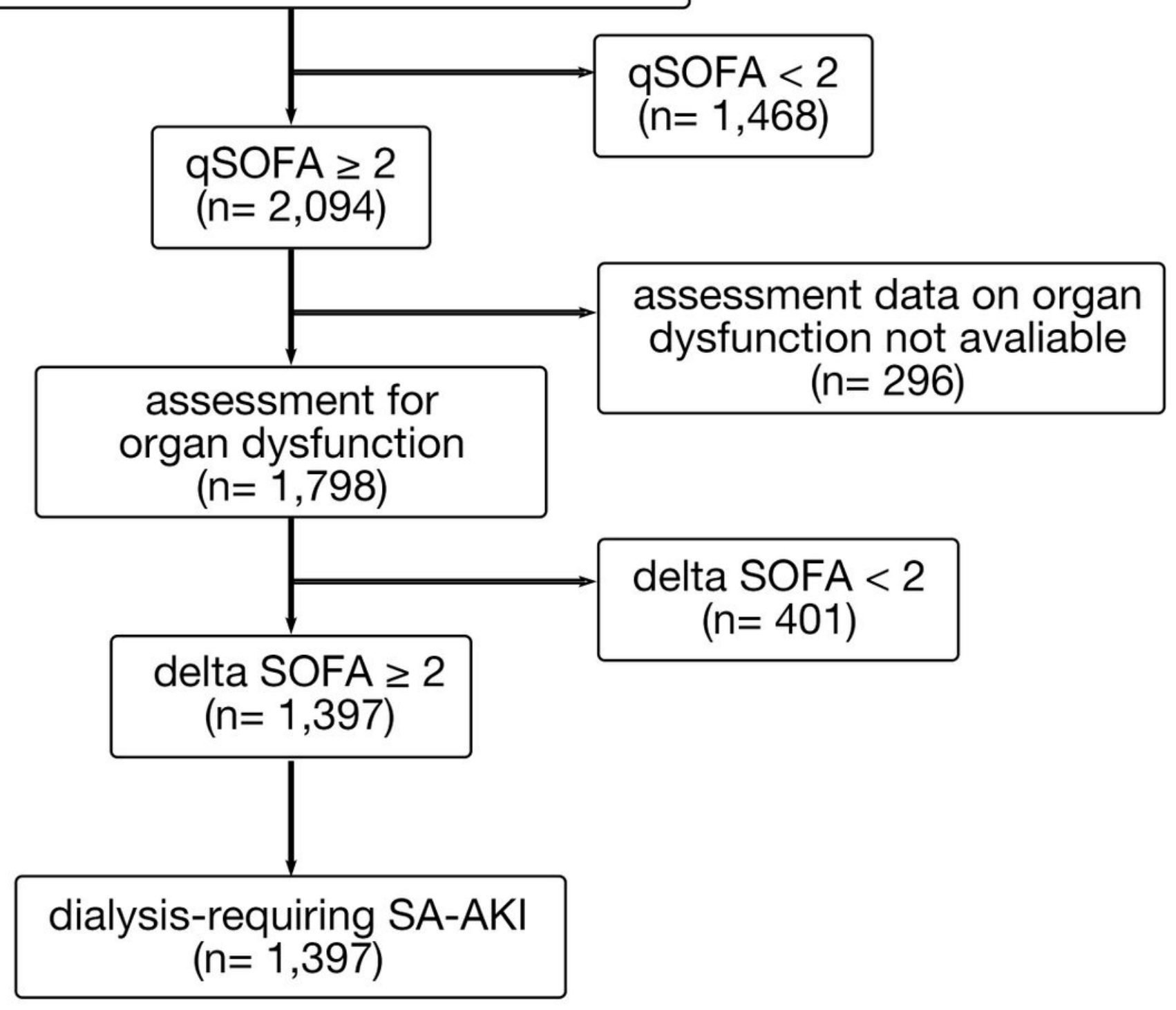

Figure 1

Flow diagram of study population. A total of 3,562 critically ill ICU patients between 1 January 2002 and 31 December 2018 were screened. In total, 1,397 patients who were diagnosed to have sepsis defined by the Sepsis-3 criteria and dialysis-requiring SA-AKI were enrolled in this study. Abbreviations: ICU, intensive care unit; AKI, acute kidney injury; SA-AKI, sepsis-associated AKI; qSOFA, quick SOFA score; SOFA, sequential organ failure assessment score. 
(A) cluster $1(n=360)$

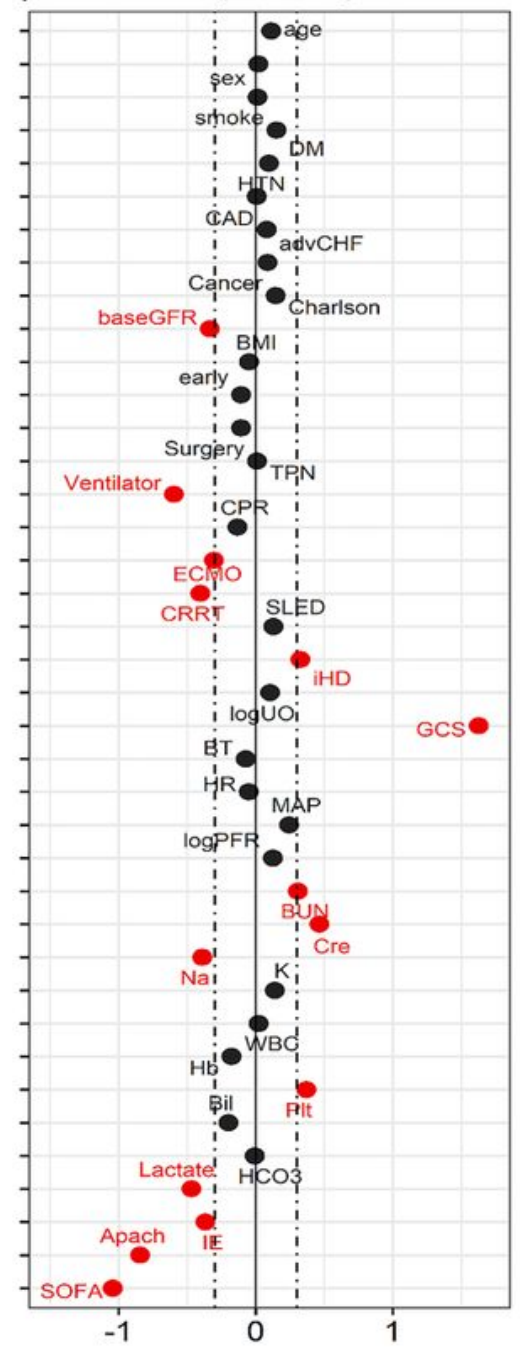

(B)

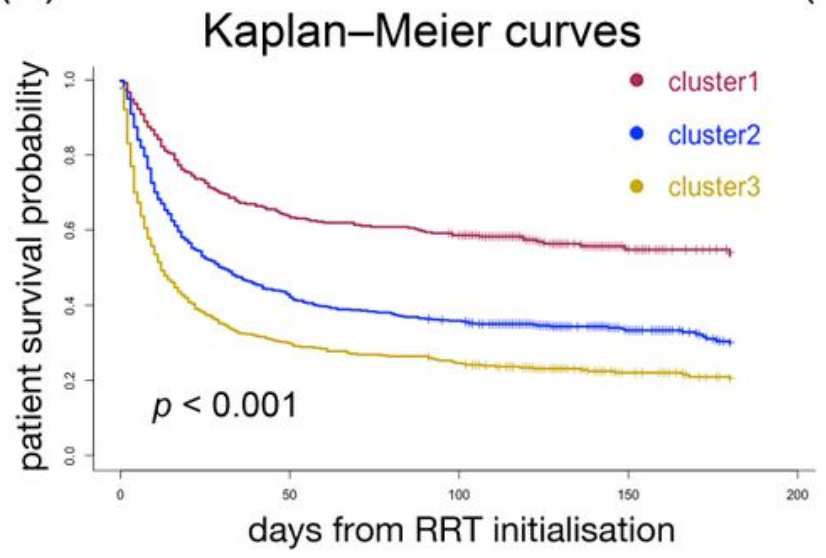

cluster $2(n=594)$

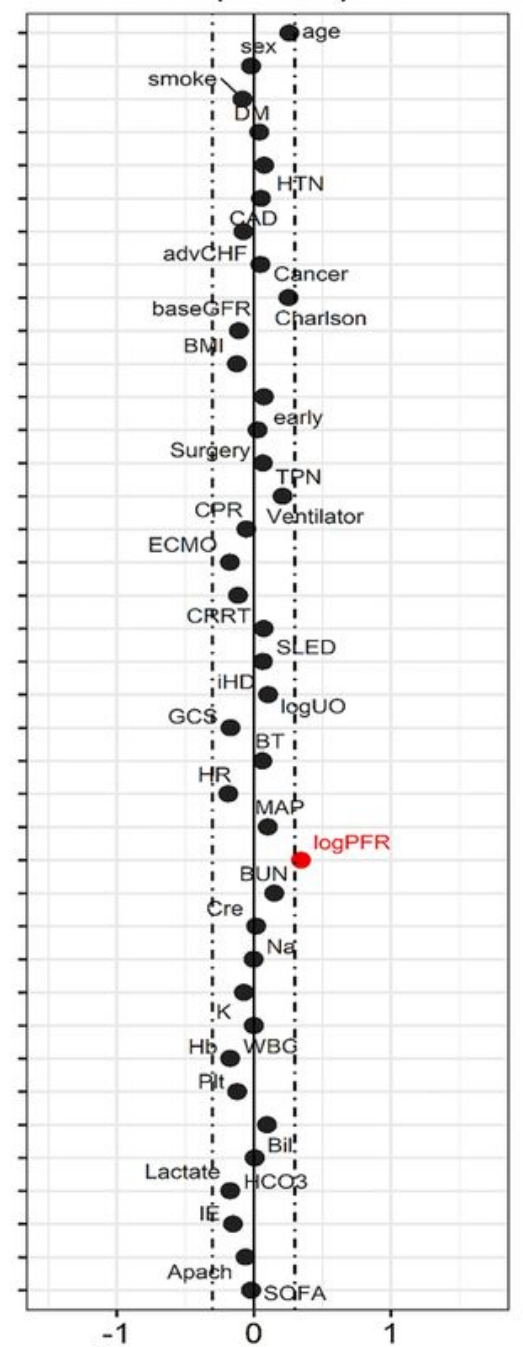

(C) cluster $3(n=443)$

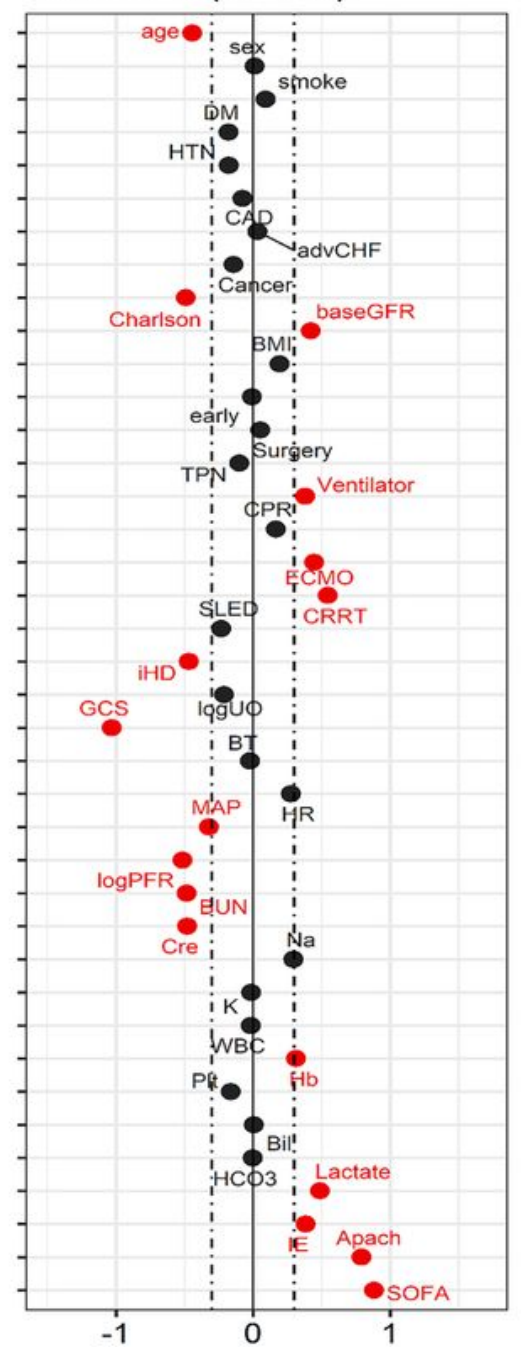

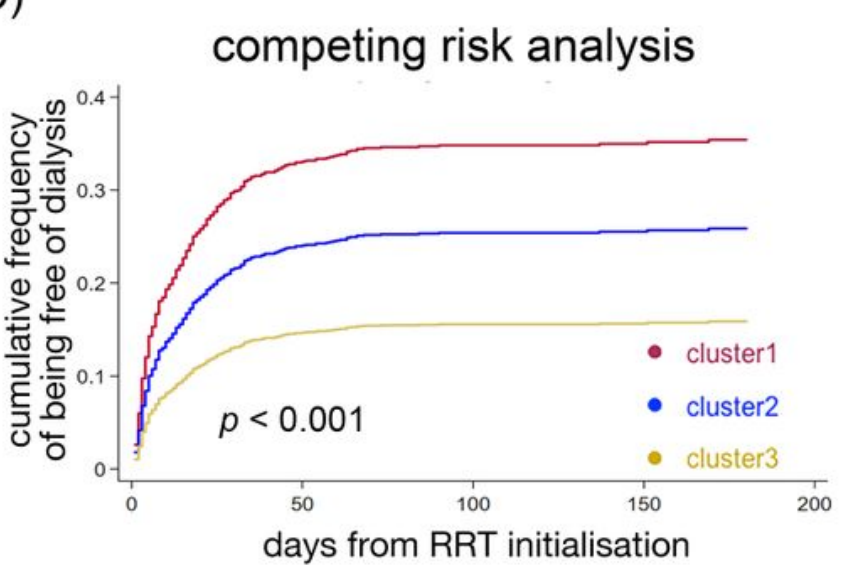

Figure 2

Baseline characteristics and outcomes across the three clusters of patients. (A) The Manhattan plot of the standardized differences in the baseline clinical characteristics across the three clusters revealed by the 22 parameters upon initialising RRT. The dashed vertical lines represent the absolute standardized difference of 0.3. Key features (having an absolute standardised difference of $\geq 0.3$ ) for each cluster are marked in red. (B) Kaplan-Meier curves depict the survival probability of patients by the three clusters. 
(C) Cumulative incidence of being free of dialysis stratified by the three clusters with death as the competing risk. Abbreviations: RRT, renal replacement therapy; DM, diabetes mellitus; HTN, hypertension; CAD, coronary artery disease; advCHF: advanced heart failure, defined by New York Heart Association functional class III or IV; Charlson, Charlson Comorbidity Index; baseGFR, baseline estimated glomerular filtration rate; $\mathrm{BMI}$, body mass index; TPN, total parenteral nutrition; CPR, cardiopulmonary resuscitation; ECMO, extracorporeal membrane oxygenation; CRRT, continuous renal replacement therapy; SLED, sustained low efficiency dialysis; iHD, intermittent haemodialysis; logUO, logarithm of urine volume; GCS, Glasgow coma scale; BT, body temperature; HR, heart rate; MAP, mean arterial pressure; logPFR, logarithm of the $\mathrm{PaO} 2$ to fraction of inspired oxygen ratio; $\mathrm{BUN}$, blood urea nitrogen; Cre, serum creatinine; $\mathrm{Na}$, sodium; K, potassium; WBC, white blood cells; Hb, hemoglobin; Plt, platelet count; Bil, total bilirubin; IE, inotropic equivalent; Apach, Acute Physiology and Chronic Health Evaluation II score; SOFA, sequential organ failure assessment score.

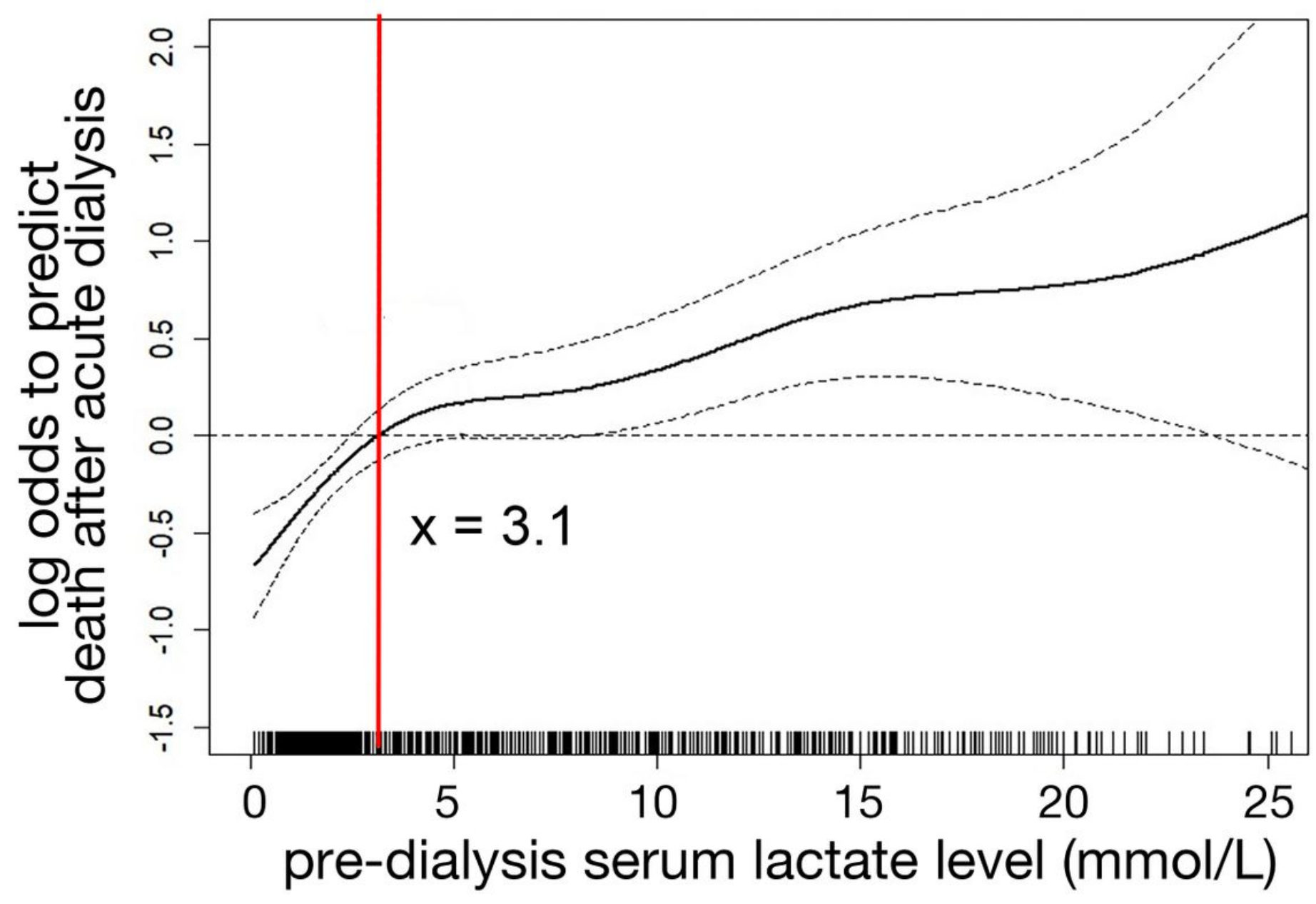

Figure 3

The probability of mortality according to the pre-dialysis serum lactate levels. The plot was drawn using a generalized additive model with adjustment for age, sex, baseline estimated glomerular filtration rate, Charlson Comorbidity Index and mean arterial pressure upon initialising RRT. The estimated probability of 
mortality 90 days after hospital discharge raised gradually accompanying the increase of serum lactate levels upon initialising renal replacement therapy.
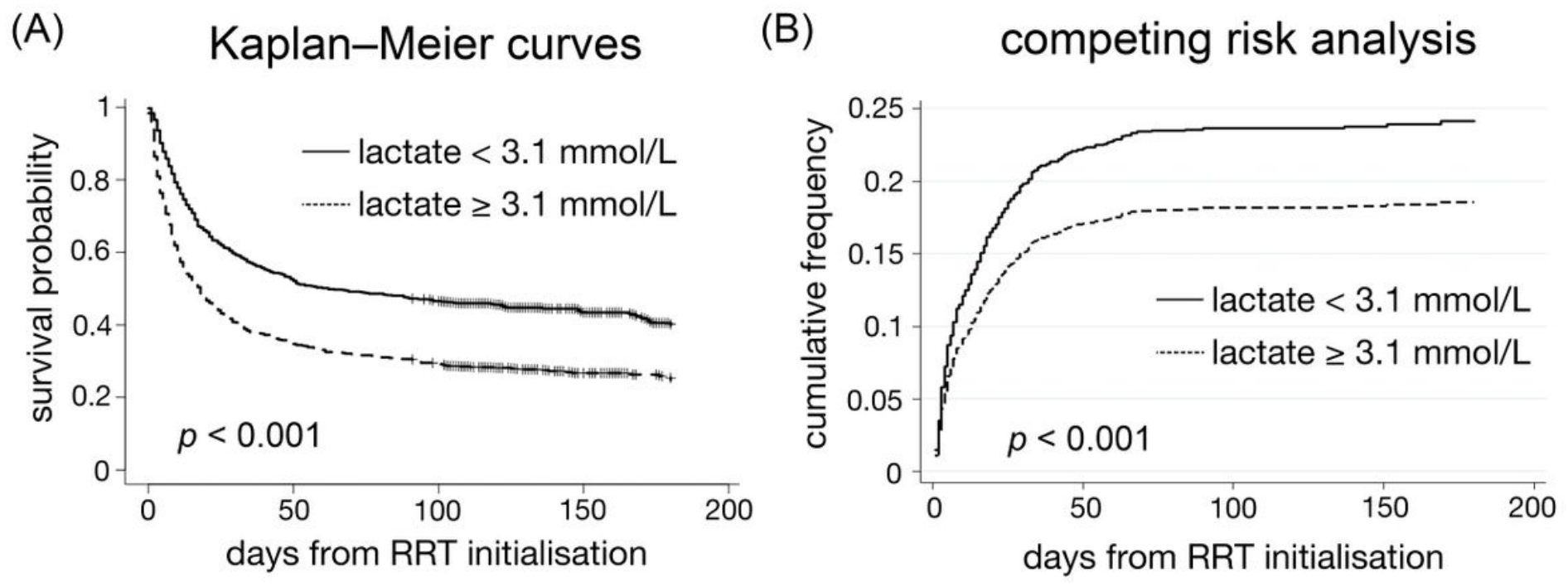

\section{Figure 4}

Associations of serum lactate level and clinical outcomes in patients with dialysis-requiring sepsisassociated acute kidney injury. (A) The Kaplan-Meier survival analysis for patients with serum lactate level $<3.1 \mathrm{mmol} / \mathrm{L}$ and $\geq 3.1 \mathrm{mmol} / \mathrm{L}$ upon initialising renal replacement therapy (RRT) (log rank test $p<$ 0.001). (B) Cumulative incidence of being free of dialysis, stratified by the threshold of serum lactate levels of $3.1 \mathrm{mmol} / \mathrm{L}$ upon initialising RRT with death as the competing risk (Gray's test $p<0.001$ ). 


\section{Kaplan-Meier curves}

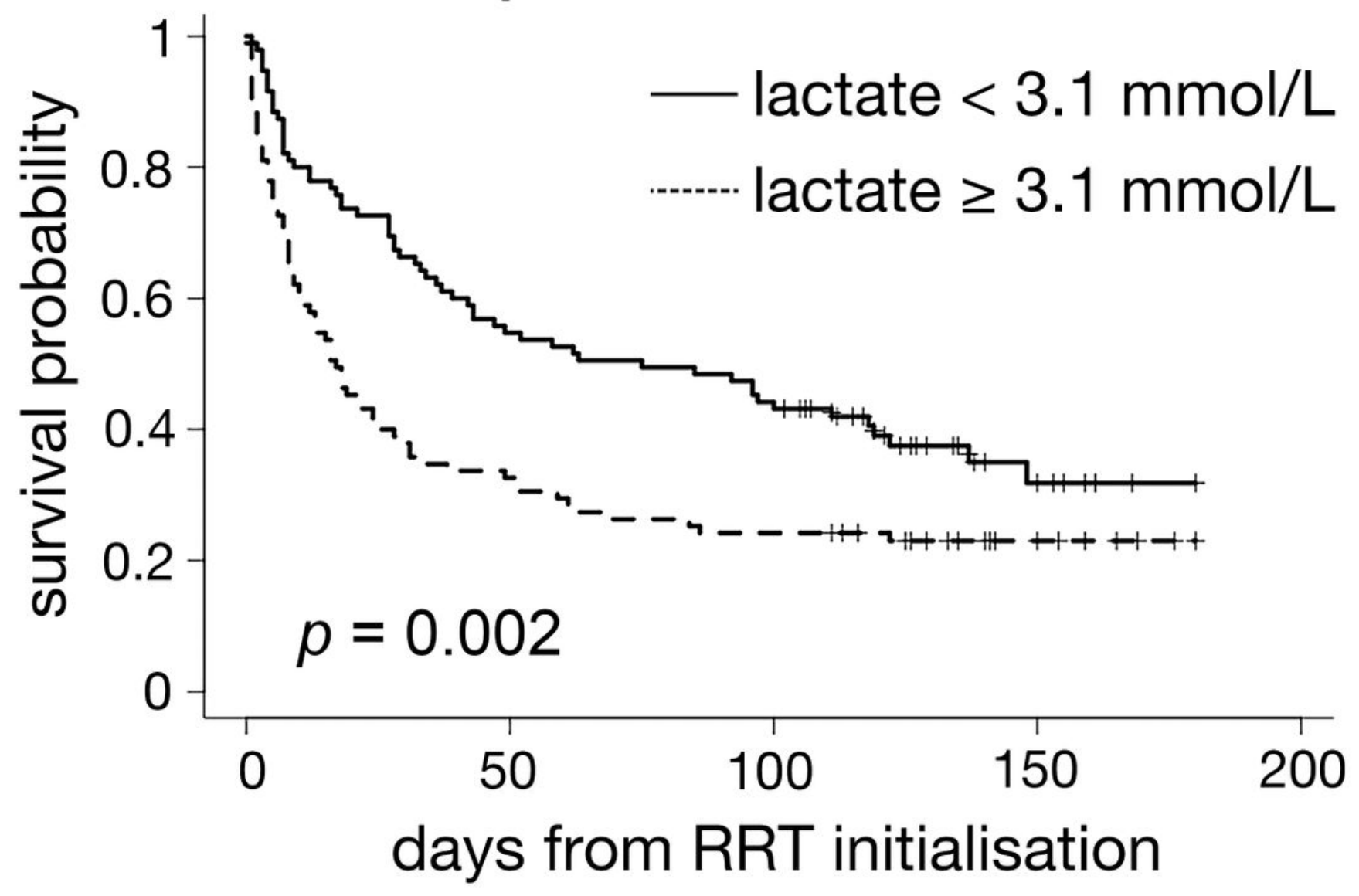

Figure 5

Survival analysis in the validation cohort. The association of hyperlactatemia with mortality was validated in 190 critically ill patients with dialysis-requiring sepsis-associated acute kidney injury from the nationwide epidemiology and prognosis of dialysis-requiring acute kidney injury study (NEP-AKI-D) cohort. Kaplan-Meier survival analysis for patients with serum lactate level $<3.1 \mathrm{mmol} / \mathrm{L}$ and $\geq 3.1$ $\mathrm{mmol} / \mathrm{L}$ upon initialising renal replacement therapy (RRT) (log rank test $\mathrm{p}=0.002)$.

\section{Supplementary Files}

This is a list of supplementary files associated with this preprint. Click to download.

- Additionalfile1.docx 